8. van Diemen CC. Postma DS. Siedlinski M. Blokstra A. Smit HA. Boezen HM. Genetic variation in TIMP1 but not MMPs predict excess FEV1 decline in two general population-based cohorts.Respir Res. 2011;12:57.)

9. Xu E. Lai M. Lv B. Xing X. Huang Q. Xia X. A single nucleotide polymorphism in the matrix metalloproteinase- 2 promoter is associated with colorectal cancer. Biochem Biophys Res Commun.2004;324:999-1003;

DOI https://doi.org/10.30525/978-9934-26-038-4-8

\title{
THE ROLE OF OVEREXPRESSION OF CYCLOOXYGENASE-2 IN THE PROGNOSIS OF INVASIVE DUCTAL BREAST CANCER
}

\author{
Volos L. I. \\ Doctor of Medical Science, \\ Professor at the Department of Pathology, \\ Danylo Halytsky Lviv National Medical University \\ Dudash A. P. \\ Ph. D. Student at Department of Pathology \\ Danylo Halytsky Lviv National Medical University, \\ Pathologist \\ Western Ukrainian Histological Laboratory \\ Lviv, Ukraine
}

Introduction. The cyclooxygenases (COX) are enzymes involved in the metabolism of prostaglandins, thromboxane and prostacyclin. Under the influence of various factors (physical, chemical, inflammatory or mitogenic stimuli), arachidonic acid is released from the phospholipids of cell membranes under the action of phospholipase A2 [1]. Under the influence of $\mathrm{COX}$, it is converted into an unstable intermediate prostaglandin $\mathrm{H} 2$ (PGH2), which under the action of tissue-specific isomerases is converted into numerous prostaglandins, thromboxane and prostacyclin. Prostaglandins activate specific receptors on cell membranes, belonging to the family of Gprotein coupled receptors. Previously two species of the enzyme COX-1 and COX-2 were identified, which are encoded by different genes. Both COX genes are very similar, as are both isoenzymes, which have almost identical three-dimensional structures. The active positions of the two COX differ in only one amino acid residue. COX-1 is constitutively expressed in most tissues and organs, including the gastrointestinal tract, kidneys and platelets 
and has a protective role in the gastrointestinal tract [2]. The COX-2 enzyme is inducible in most tissues in response to inflammatory and hormonal signals. COX-2 is expressed constitutively in the brain and in the cortex of the kidneys. In other tissues, COX-2 expression is pathological and is induced by stimulating factors such as cytokines, free oxygen radicals, lipopolysaccharides, tissue plasminogen activators, mitogenic factors [3]. Recent studies have indicated that the overexpression of COX-2 is associated with cancers of the stomach, colon, bladder and prostate and it was suggested that COX-2 may be involved in tumorigenesis in various organs [4-6]. In breast cancer, a high level of prostaglandin and COX-2 expression was reported in breast cancer tissue, but studies on the role of COX-2 in the development and the progression of breast cancer are relatively few [7-11].

The aim of this study was to determine of COX-2 expression and correlation with clinicopathologic prognostic parameters in the progression of invasive ductal breast cancer.

Materials and methods. One hundred and ninety-three patients who underwent surgery for breast cancer without neoadjuvant chemotherapy in 2017 at the Lviv State Oncological Regional Treatment and Diagnostic Center were examined. Pathological evaluation was performed using stained hematoxylin-eosin-stained sections. The histologic type of the breast cancer was determined according to the WHO classification. The histologic grade was categorized as grade I, grade II and grade III using the Elston-modified Bloom and Richardson grading system [8]. The TNM was used for clinical staging of breast cancer and clinicopathologic characteristics included tumor size, lymph node status, stage, ER, PR, Her2/neu status, and histologic grades were evaluated. A total of 193 cases of breast cancer, using immunohistochemistry, were classified into four major molecular subtypes (luminal A, luminal B, HER2/neu-positive, and triple negative). A monoclonal antibody to COX-2 (Clone SP21, Thermo scientific), ER (Clone EP1, Dako), PR (PgR 636, Dako Flex), Her2-neu (Clone SP3 Thermo scientific) and streptavidin peroxidase detection system with diaminobenzidine tetrachloride were used. The immunoreactive score was calculated for ER, PR and Her$2 /$ neu by multiplication of the percentage of positive cells and the staining intensity. The percentage of positive cells was categorized as 0 (negative), 1 (10\% positive cells), 2 (10-50\% positive cells), 3 (51-80\% positive cells) and $4(80 \%$ positive cells). The staining intensity was categorized as 0 (negative), 1 (weak), 2 (moderate) and 3 (strong). For COX-2 the results of immunohistochemistry were interpreted as positive when $10 \%$ of the tumor cells demonstrated clear cytoplasmic staining or an immunoreactive score of 2 was considered positive. Chi-squared test was performed to evaluate the relationship between these subtypes and clinicopathological features. 
Results. Among the 193 invasive ductal breast cancer cases, 79 (40,9\%) patients with luminal A subtype, $32(16,6 \%)$ revealed a triple-negative phenotype (TNBC), 39 (20,2\%) patients with HER-2/neu positive (non-luminal) and $43(22,3 \%)$ with Luminal B invasive breast carcinoma.

79 patients with luminal A subtype were identified by immunohistochemistry (IHC): ER+, PR+, HER2- and Ki-67 less than 20 percent on surgically resected breast cancer tissue. Nottingham Histologic Grade distribution was as follows: G1 - $10(12,66 \%), \mathrm{G} 2-56(70,88 \%)$, and G3 $13(16,46 \%)$ cases and clinical stage II - $35(44,30 \%)$ and III - $31(39,24 \%)$ was observed. The patients with Luminal A subtype had a median age of 50 years (range, $32-85$ years), 25 (31,6\%) were under 55 years and menopausal status was in $68,4 \%$ of cases.

The 32 patients with TNBC had a median age of 59 years (range, 31-79 years), $11(34,4 \%)$ were under 55 years. Clinical stage II $14(43,74 \%)$ and III - $11(34,38 \%)$ was observed. Nottingham Histologic Grade distribution was as follows: G1 - $0(0 \%), \mathrm{G} 2-17(53,13 \%)$, and G3 $14(43,74 \%), \mathrm{G} 4-1(3,13 \%)$ cases. Menopausal status was in $65,6 \%$ of cases. Unilateral lesion of the breast was detected in most cases and tumors were more frequently localized in the outer quadrants $(62,5 \%)$.

The median age of the 39 patients with HER-2/neu positive (non-luminal) invasive ductal breast cancer was 50,5 years with range $28-78$ years and $21(56,8 \%)$ were under 55 years, 20 of $37(54,1 \%)$ patients were premenopausal at the time of surgery.

The 43 patients with Luminal B invasive ductal breast cancer had a median age of 49 years (range, 27-73 years), $22(51,2 \%)$ were under 55 years. Clinical stage II - $23(53,49 \%)$ and III - $10(23,25 \%)$ was observed. Nottingham Histologic Grade distribution was as follows: G1 - 5 (11,62\%), G2 - $27(62,79 \%)$, and G3 - $11(25,58 \%)$ cases. Menopausal status was in $48,8 \%$ of cases.

COX-2 was positive in 70 of 102 cases $(69 \%)$ in Stage II, and 52 of 61 cases $(85 \%)$ in Stage III, which was statistically significant $(\mathrm{p}<0,05)$. Of the 193 cases of invasive carcinoma, COX-2 was positive in 31 of 47 cases $(65 \%)$ measuring $2 \mathrm{~cm}$, in 85 of 103 cases $(82 \%)$ measuring $2 \mathrm{~cm}$ and $5 \mathrm{~cm}$, and all 43 cases $(100 \%)$ measuring $5 \mathrm{~cm}$, which was statistically significant also ( $<<0,01)$. Cases with positive ER and PR tended to more frequently overexpress COX-2, but there was no significant correlation. Histologic grade was not correlated to cyclooxygenase- 2 overexpression.

In all cases C-erbB-2 staining was scored 3 and considered as positive for HER-2/neu overexpression. In the present study, we found that COX-2 was expressed in all HER-2/neu positive tumours, but overexpression associated with parameters of the poor prognosis: postmenopausal status $(n=10 ; 62,5 \%)$, large tumour size $(n=25 ; 67,6 \%)$, tumour higher grade 
(G2 - n=27; 73\%; G3 - n=9; 24,3\%) and lymph node metastasis $(\mathrm{n}=13 ; 35,1 \%)(\mathrm{p}<0,05)$.

Conclusions. Our results demonstrated COX-2 positivity in $82 \%$ of invasive ductal breast cancer and COX-2 overexpression was significantly correlated with aggressive phenotypic features, such as HER-2/neu overexpression and high histological grade, large tumor size, advanced stage. Increased expression of COX-2 in tissue of invasive ductal breast cancer could indicate a role in breast carcinogenesis. The results indicate that COX-2 may contribute to the development of breast cancer and can be used as a prognostic clinically biomarker for estimating tumor aggressiveness.

\section{References:}

1. Dubois RN, Abramson SB, Crofford L et al. Cyclooxygenase in biology and disease. FASEB J. 1998; 12; 1063-1073.

2. Williams CS, Mann M, Dubois RN. The role of cyclooxygenases in inflammation, cancer, and development. Oncogene 1999; 18; 7908-7916.

3. . Jones DA, Carlton DP, McIntyre TM, Zimmerman GA, Prescott SM. Molecular cloning of human prostaglandin endoperoxide synthase type II and demonstration of expression in response to cytokines. J Biol Chem 1993; 268: 9049-54.

4. Ristimaki A, Honkanen N, Jankala H, Sipponen P, Harkonen M. Expression of cyclooxygenase-2 in human gastric carcinoma. Cancer Res 1997; 57:1276-80.

5. Yoshimura R, Sano H, Masuda C, Kawamura M, Tsubouchi Y, Chargui $\mathrm{J}$, et al. Expression of cyclooxygenase-2 in prostate carcinoma. Cancer 2000; 89:589-96.

6. Mohammed SU, Knapp DW, Bostwick DG, Foster RS, Khan KN, Masferrer JL, et al. Expression of cyclooxygenase-2 (COX-2) in human invasive transitional cell carcinoma (TCC) of the urinary bladder. Cancer Res 1999; 59:5647-50.

7. Parrett M, Harris RE, Joarder FS, Ross MS, Clausen KP, Robertson FM. Cyclooxygenase-2 gene expression in human breast cancer. Int J Oncol 1997; 10:503-7.

8. Elston CW, Ellis IO. Pathological prognostic factors in breast cancer. I. The value of histological grade in breast cancer: experience from a large study with long-term follow-up. Histopathology 1991; 5:403-10.

9. Witton CJ, Hawe S J K, Cooke TG, Bartlett J M S. Cyclooxygenase 2 (COX2) expression is associated with poor outcome in ER-negative, but not ER-positive, breast cancer. Histopathology. 2004; 45: 47-54.

10. Jana D, Sarkar D, Ganguly S, et al. Role of cyclooxygenase 2 (COX2) in prognosis of breast cancer. Indian J Surg Oncol. 2014; 5:59-65. 
11. Solanki R, Agrawal N, Ansari M, Jain S, Jindal A. COX-2 Expression in Breast Carcinoma with Correlation to Clinicopathological Parameters. Asian Pac J Cancer Prev. 2018 Jul 27;19(7):1971-1975.

DOI https://doi.org/10.30525/978-9934-26-038-4-9

\title{
QUANTITATIVE AND QUALITATIVE INDICATORS OF BLOOD FLOW THROUGH THE MAIN ARTERIES OF THE HEAD IN PATIENTS WITH STROKE
}

\author{
Hryhorova I. A. \\ Doctor of Medical Sciences, Professor, \\ Head of the Department Neurology № 1 \\ Kharkiv National Medical University \\ Tykhonova L. V. \\ Candidate of Medical Sciences, \\ Associate professor at the Department of Neurology № 1 \\ Kharkiv National Medical University \\ Ibrahimova O. L. \\ Candidate of Medical Sciences, \\ Assistant Professor at the Department of Neurology № 1 \\ Kharkiv National Medical University \\ Kharkiv, Ukraine
}

Stenosis and blockage of the arteries of the vertebrobasilar basin of the VBB, which are most often caused by atherosclerosis and atherothrombosis are manifested by a wide range of clinical conditions. The lesion of the VBB attracts the attention not only of neurologists, but also of emergency physicians, neurosurgeons, neuroradiologists and rehabilitologists, as patients with this pathology often find it difficult to establish an accurate diagnosis, assess prognosis and choose optimal treatment and rehabilitation measures. Many aspects of the diagnosis and treatment of vascular lesions of the VBB remain insufficiently studied [1-3]. Every year, more than 1.2 million people in Europe suffer from cerebral stroke (CS) and World Health Organization experts predict a further increase in the incidence. Cerebral stroke is the leading cause of disability and mortality throughout the world [5].

The aim of our study is to improve the diagnostic algorithm for ischemic stroke. To identify the pathogenetic subtype of ischemic stroke, as an addi- 